\title{
T-violation search with very long baseline neutrino oscillation experiments
}

\author{
Masafumi Koike* \\ Institute for Cosmic Ray Research, University of Tokyo, Midori-cho, Tanashi, Tokyo 188-8502, Japan \\ and \\ Joe $\mathrm{Sato}^{\dagger}$ \\ Research Center for Higher Education, Kyushu University, Ropponmatsu, Chuo-ku, Fukuoka 810-8560, Japan
}

\begin{abstract}
We consider possibilities of observing T-violation effects in neutrino oscillation experiments with very long baseline $(\sim 10000 \mathrm{~km})$ using low energy neutrino $(\sim$ several hundreds $\mathrm{MeV}$ ). We show that the matter effect effectively changes only the first-second generation mixing angle, respecting solar neutrino deficits and atmospheric neutrino anomalies. The effective mixing of the first-second generation in the Earth grows up to maximum by resonance. This effect enables one to search $\mathrm{T}$ violation in case the first-second mixing angle is small. We discuss its implications to the observations of T-violation effects in long baseline experiments.
\end{abstract}

\section{Introduction}

One of the most important questions in particle physics is whether neutrinos are massive and if so whether there is a mixing among leptons. Many experiments and observations have shown evidences for neutrino oscillation one after another, which indicates that neutrinos are massive and there is a mixing in lepton sector[1].

*e-mail address: koike@icrr.u-tokyo.ac.jp

$\dagger$ e-mail address: joe@rc.kyushu-u.ac.jp 
The solar neutrino deficit has long been observed [2, 3, 4, 5, 6]. The atmospheric neutrino anomaly has been found[4, 8, 9, 10] and recently almost confirmed by SuperKamiokande[11]. There is also another suggestion given by LSND[12]. All of them can be understood by neutrino oscillation.

Since there is a mixing in lepton sector, it is quite natural to imagine that there occurs CP violation in lepton sector. Several physicists have considered whether we may see CP-violation effect in lepton sector through long baseline neutrino oscillation experiments. First it has been studied in the context of currently planed experiments [13, 14, 15, 16, 17, 18, 19, 20] and recently in the context of neutrino factory 21, 22, 23, 24.

In this paper we consider very long baseline experiments $(\sim 10000 \mathrm{~km})$ with low energy neutrinos $(\sim$ several hundreds $\mathrm{MeV})[25,[26]$. We have great advantages to use them to see $\mathrm{CP}$ violation effect in long baseline experiments:

1. Availability of incident $\nu_{\mathrm{e}}\left(\bar{\nu}_{\mathrm{e}}\right)$ beam.

T-conjugate channel can be compared in long baseline neutrino oscillation experiments by use of electron neutrinos. It enables to search "matter-effectfree" CP-violation effect.

2. Good energy resolution and well calculated flux.

This is also important. We need to know neutrino flux very precisely since CPviolation effect may be a small effect. Clear experimental signals are needed.

3. Availability of low energy neutrinos.

The most important point. At least three kinds of neutrinos must play an important role in neutrino oscillation to search CP-violation effect since it arises as three(or more)-generation phenomena [27, 28]. We have to use low energy neutrinos to make them play the game.

Thus it is of great interest to consider the possibility to observe $\mathrm{CP}$ violation in long baseline experiments with low energy (several hundreds $\mathrm{MeV}$ ) neutrinos [26].

Experimentally, the observation of solar neutrino allows two regions of mixing angle [31], usually referred to as large (MSW[29, 30]) and small (MSW \& Vacuum) mixing angle solution. It seems difficult to see $\mathrm{CP}$ violation for the small mixing angles, since in such a case CP-violation effect is also in general small. It is worthwhile to investigate the possibility of $\mathrm{CP}$ violation search in case the mixing angle is small.

From this viewpoint we consider in this paper possibilities of observing CPviolation effects in neutrino oscillation experiments with very long baseline $(\sim 10000$ $\mathrm{km})$ using low energy neutrino $(\sim$ several hundreds $\mathrm{MeV})$. We focus our attention to "T-violation" channel[32, 33, 34]. Three generations of neutrinos without any sterile ones are assumed, with solar neutrino deficit and atmospheric neutrino anomaly attributed to the neutrino oscillation.

\section{Mixing matrix and mass in the Earth}

We first derive here the mixing matrix and mass squared in the Earth for neutrinos with low energy (several hundreds $\mathrm{MeV}$ ). assuming three generations of active 
neutrinos without any sterile one.

Let the mass eigenvalues of three neutrinos be $m_{i}(i=1,2,3)$ and MNS mixing matrix 37 $U$ which relates the flavor eigenstates $\nu_{\alpha}(\alpha=\mathrm{e}, \mu, \tau)$ and the mass eigenstates in the vacuum $\nu_{i}^{\prime}(i=1,2,3)$ as

$$
\nu_{\alpha}=U_{\alpha i} \nu_{i}^{\prime} \text {. }
$$

We parametrize $U$ [38, 32, 34] with the Gell-Mann matrices $\lambda_{i}$ 's as

$$
\begin{aligned}
& U=\mathrm{e}^{\mathrm{i} \psi \lambda_{7}} \Gamma \mathrm{e}^{\mathrm{i} \phi \lambda_{5}} \mathrm{e}^{\mathrm{i} \omega \lambda_{2}} \\
= & \left(\begin{array}{ccc}
1 & 0 & 0 \\
0 & c_{\psi} & s_{\psi} \\
0 & -s_{\psi} & c_{\psi}
\end{array}\right)\left(\begin{array}{ccc}
1 & 0 & 0 \\
0 & 1 & 0 \\
0 & 0 & \mathrm{e}^{\mathrm{i} \delta}
\end{array}\right)\left(\begin{array}{ccc}
c_{\phi} & 0 & s_{\phi} \\
0 & 1 & 0 \\
-s_{\phi} & 0 & c_{\phi}
\end{array}\right)\left(\begin{array}{ccc}
c_{\omega} & s_{\omega} & 0 \\
-s_{\omega} & c_{\omega} & 0 \\
0 & 0 & 1
\end{array}\right) \\
= & \left(\begin{array}{ccc}
c_{\phi} c_{\omega} & c_{\phi} s_{\omega} & s_{\phi} \\
-c_{\psi} s_{\omega}-s_{\psi} s_{\phi} c_{\omega} \mathrm{e}^{\mathrm{i} \delta} & c_{\psi} c_{\omega}-s_{\psi} s_{\phi} s_{\omega} \mathrm{i} \mathrm{e}^{\mathrm{i} \delta} & s_{\psi} c_{\phi} \mathrm{e}^{\mathrm{i} \delta} \\
s_{\psi} s_{\omega}-c_{\psi} s_{\phi} c_{\omega} \mathrm{e}^{\mathrm{i} \delta} & -s_{\psi} c_{\omega}-c_{\psi} s_{\phi} s_{\omega} \mathrm{e}^{\mathrm{i} \delta} & c_{\psi} c_{\phi} \mathrm{e}^{\mathrm{i} \delta}
\end{array}\right),
\end{aligned}
$$

where $c_{\psi}=\cos \psi, s_{\phi}=\sin \phi$, etc.

The evolution equation of the flavor eigenstate of neutrinos $\nu=\left(\nu_{\mathrm{e}}, \nu_{\mu}, \nu_{\tau}\right)^{\mathrm{T}}$ that has energy $E$ in matter with electron density $n_{\mathrm{e}}$ is given by

$$
-\mathrm{i} \frac{\mathrm{d} \nu}{\mathrm{d} x}=H \nu
$$

where

$$
\begin{aligned}
H & =U\left(\begin{array}{lll}
0 & & \\
& \delta m_{21}^{2} & \\
& & \delta m_{31}^{2}
\end{array}\right) U^{\dagger}+\left(\begin{array}{lll}
a & & \\
& 0 & \\
& & 0
\end{array}\right) \\
& =\tilde{U}\left(\begin{array}{ccc}
\tilde{m}_{1}^{2} & & \\
& \tilde{m}_{2}^{2} & \\
& & \tilde{m}_{3}^{2}
\end{array}\right) \tilde{U}^{\dagger} .
\end{aligned}
$$

Here

$$
a=2 \sqrt{2} G_{\mathrm{F}} n_{\mathrm{e}} E=3.04 \times 10^{-4} \mathrm{eV}^{2} \cdot\left(\frac{E}{\mathrm{GeV}}\right)\left(\frac{\rho}{4.46 \mathrm{~g} \cdot \mathrm{cm}^{-3}}\right)
$$

is a factor due to effective mass in the matter.

The solar neutrino deficit implies $\delta m^{2} \sim O\left(10^{-4} \sim 10^{-5}\right) \mathrm{eV}^{2}$, while the atmospheric neutrino anomaly suggests $\delta m^{2} \sim O\left(10^{-2} \sim 10^{-3}\right) \mathrm{eV}^{2}$. We regard them as $\delta m_{21}^{2}$ and $\delta m_{31}^{2}$ respectively. With these values and eq.(河) we assume $a, \delta m_{21}^{2} \ll \delta m_{31}^{2}$ and diagonalize eq. (雨) up to the lowest order. Letting

$$
\begin{aligned}
H^{\prime} & \equiv \mathrm{e}^{\mathrm{i} \omega \lambda_{2}} U^{\dagger} H U \mathrm{e}^{-\mathrm{i} \omega \lambda_{2}} \\
& =\left(\begin{array}{ccc}
0 & & \\
& 0 & \\
& \delta m_{31}^{2}
\end{array}\right) \\
& +\mathrm{e}^{\mathrm{i} \omega \lambda_{2}}\left(\begin{array}{ccc}
0 & & \\
& \delta m_{21}^{2} & \\
& & 0
\end{array}\right) \mathrm{e}^{-\mathrm{i} \omega \lambda_{2}}+\mathrm{e}^{\mathrm{i} \omega \lambda_{2}} U^{\dagger}\left(\begin{array}{lll}
a & & \\
& 0 & \\
& & 0
\end{array}\right) U \mathrm{e}^{-\mathrm{i} \omega \lambda_{2}} \\
& =H_{0}^{\prime}+H_{1}^{\prime},
\end{aligned}
$$


where

$$
H_{0}^{\prime}=\left(\begin{array}{ccc}
0 & & \\
& 0 & \\
& & \delta m_{31}^{2}
\end{array}\right)
$$

and

$$
\begin{aligned}
H_{1}^{\prime} & =\mathrm{e}^{\mathrm{i} \omega \lambda_{2}}\left(\begin{array}{ccc}
0 & & \\
& \delta m_{21}^{2} & \\
& & 0
\end{array}\right) \mathrm{e}^{-\mathrm{i} \omega \lambda_{2}}+\mathrm{e}^{\mathrm{i} \omega \lambda_{2}} U^{\dagger}\left(\begin{array}{ccc}
a & & \\
& 0 & \\
& & 0
\end{array}\right) U \mathrm{e}^{-\mathrm{i} \omega \lambda_{2}} \\
& =\delta m_{21}^{2}\left(\begin{array}{cccc}
s_{\omega}^{2} & c_{\omega} s_{\omega} & 0 \\
c_{\omega} s_{\omega} & c_{\omega}^{2} & 0 \\
0 & 0 & 0
\end{array}\right)+a\left(\begin{array}{ccc}
c_{\phi}^{2} & 0 & c_{\phi} s_{\phi} \\
0 & 0 & 0 \\
c_{\phi} s_{\phi} & 0 & c_{\phi}^{2}
\end{array}\right),
\end{aligned}
$$

we diagonalize $H^{\prime}$ by perturbation. $H_{0}^{\prime}$ has degenerate eigenvalues, so we first diagonalize

$$
\begin{aligned}
h_{1}^{\prime} & \equiv\left(\begin{array}{cc}
a c_{\phi}^{2}+\delta m_{21}^{2} s_{\omega}^{2} & \delta m_{21}^{2} c_{\omega} s_{\omega} \\
\delta m_{21}^{2} c_{\omega} s_{\omega} & \delta m_{21}^{2} c_{\omega}^{2}
\end{array}\right) \\
& =\frac{a c_{\phi}^{2}+\delta m_{21}^{2}}{2}+\frac{1}{2}\left(\begin{array}{cc}
a c_{\phi}^{2}-\delta m_{21}^{2} c_{2 \omega} & \delta m_{21}^{2} s_{2 \omega} \\
\delta m_{21}^{2} s_{2 \omega} & -a c_{\phi}^{2}+\delta m_{21}^{2} c_{2 \omega}
\end{array}\right) .
\end{aligned}
$$

The eigenvalues of (10) are

$$
\lambda_{ \pm}=\frac{a c_{\phi}^{2}+\delta m_{21}^{2}}{2} \pm \frac{1}{2} \sqrt{\left(a c_{\phi}^{2}-\delta m_{21}^{2} c_{2 \omega}\right)^{2}+\left(\delta m_{21}^{2}\right)^{2} s_{2 \omega}^{2}}
$$

so that $h_{1}^{\prime}$ is diagonalized as

$$
h_{1}^{\prime}=\left(\begin{array}{cc}
\cos \tilde{\omega} & \sin \tilde{\omega} \\
-\sin \tilde{\omega} & \cos \tilde{\omega}
\end{array}\right)\left(\begin{array}{cc}
\lambda_{-} & 0 \\
0 & \lambda_{+}
\end{array}\right)\left(\begin{array}{cc}
\cos \tilde{\omega} & -\sin \tilde{\omega} \\
\sin \tilde{\omega} & \cos \tilde{\omega}
\end{array}\right)
$$

where $\tilde{\omega}$ satisfies

$$
\begin{aligned}
\tan 2 \tilde{\omega} & =\frac{\delta m_{21}^{2} s_{2 \omega}}{-a c_{\phi}^{2}+\delta m_{21}^{2} c_{2 \omega}} \\
\sin 2 \tilde{\omega} & =\frac{\delta m_{21}^{2} s_{2 \omega}}{\sqrt{\left(a c_{\phi}^{2}-\delta m_{21}^{2} c_{2 \omega}\right)^{2}+\left(\delta m_{21}^{2} s_{2 \omega}\right)^{2}}} .
\end{aligned}
$$

The eigenvalues of $H^{\prime}$ (and thus of $H$ ) to the first order are thus

$$
\begin{aligned}
& \tilde{m}_{1}^{2}=\frac{a c_{\phi}^{2}+\delta m_{21}^{2}}{2}-\frac{1}{2} \sqrt{\left(a c_{\phi}^{2}-\delta m_{21}^{2} c_{2 \omega}\right)^{2}+\left(\delta m_{21}^{2}\right)^{2} s_{2 \omega}^{2}} \\
& \tilde{m}_{2}^{2}=\frac{a c_{\phi}^{2}+\delta m_{21}^{2}}{2}+\frac{1}{2} \sqrt{\left(a c_{\phi}^{2}-\delta m_{21}^{2} c_{2 \omega}\right)^{2}+\left(\delta m_{21}^{2}\right)^{2} s_{2 \omega}^{2}}
\end{aligned}
$$

and

$$
\tilde{m}_{3}^{2}=\delta m_{31}^{2}+a s_{\phi}^{2}
$$


We finally obtain at the lowest order

$$
H=\left[U \mathrm{e}^{-\mathrm{i}(\omega-\tilde{\omega}) \lambda_{2}}\right] \cdot \operatorname{diag}\left(\tilde{m}_{1}^{2}, \tilde{m}_{2}^{2}, \tilde{m}_{3}^{2}\right) \cdot\left[U \mathrm{e}^{-\mathrm{i}(\omega-\tilde{\omega}) \lambda_{2}}\right]^{\dagger} .
$$

Note that (14) has resonant peak at $\tilde{\omega}=\pi / 4$ or

$$
a c_{\phi}^{2}=\delta m_{21}^{2} c_{2 \omega}
$$

This condition can be written in terms of neutrino energy $E$ as

$$
E=E_{\text {peak }} \equiv 294 \mathrm{MeV} \times \frac{c_{2 \omega}}{c_{\phi}^{2}}\left(\frac{\delta m_{21}^{2}}{10^{-4} \mathrm{eV}^{2}}\right)\left(\frac{\rho}{4.46 \mathrm{~g} \cdot \mathrm{cm}^{-3}}\right)^{-1} .
$$

It is quite interesting that $\sin 2 \tilde{\omega}$ grows up to maximum value of 1 when $E$ has certain value determined by eq.(19) or eq.(20), regardless how small $\omega$ itself may be. We persue in the next section the possible merit of this enhancement in the CP or $\mathrm{T}$ violation search with the long baseline neutrino oscillation experiments.

\section{T-violation search in the very long baseline experiments}

\subsection{Resonance of T-violation effect}

The factor

$$
U \mathrm{e}^{-\mathrm{i}(\omega-\tilde{\omega})}=\mathrm{e}^{\mathrm{i} \psi \lambda_{7}} \Gamma \mathrm{e}^{\mathrm{i} \phi \lambda_{5}} \mathrm{e}^{\mathrm{i} \tilde{\omega} \lambda_{2}},
$$

which appears in eq.(18), is different from $U$ in eq.(2) only by a simple replacement $\omega \rightarrow \tilde{\omega}$. So the matter effect is included in the lowest order by the substitution $\delta m_{i j}^{2} \rightarrow \delta \tilde{m}_{i j}^{2} \equiv \tilde{m}_{i}^{2}-\tilde{m}_{j}^{2}$ and $\omega \rightarrow \tilde{\omega}$. The T-violation effect in the presence of matter is thus given in this order by

$$
P\left(\nu_{\mu} \rightarrow \nu_{\mathrm{e}}\right)-P\left(\nu_{\mathrm{e}} \rightarrow \nu_{\mu}\right)=4 \tilde{J}\left(\sin \frac{\delta \tilde{m}_{21}^{2} L}{2 E}+\sin \frac{\delta \tilde{m}_{32}^{2} L}{2 E}+\sin \frac{\delta \tilde{m}_{13}^{2} L}{2 E}\right)
$$

where

$$
\begin{aligned}
\tilde{J} & =\cos ^{2} \phi \sin \phi \cos \psi \sin \psi \cos \tilde{\omega} \sin \tilde{\omega} \sin \delta \\
& =\frac{1}{4} \cos ^{2} \phi \sin \phi \sin 2 \psi \sin 2 \tilde{\omega} \sin \delta
\end{aligned}
$$

is the effective Jarlskog parameter in matter. We have already stated at the end of previous section that $\sin 2 \tilde{\omega}$ has resonant peak at certain energy. $\tilde{J}$ has indeed this factor, so the T-violation effect is expected to have resonant peak.

With this prospect we present in Fig.1 and Fig.2 the T-violation effect averaged over energy resolution (here tentatively taken as $30 \mathrm{MeV}$ ) as a function of energy $E$. Both the perturbed result and the full calculation result are shown to exhibit how 
well our approximation works. The parameters not shown in the figure are fixed as an example to

$$
\delta m_{31}^{2}=1 \times 10^{-3} \mathrm{eV}^{2}, \psi=\frac{\pi}{4}, L=10000 \mathrm{~km}, \quad \text { and } \quad \rho=4.46 \mathrm{~g} / \mathrm{cm}^{-3} .
$$

$L$ is taken to $10000 \mathrm{~km}$ since it must be the same order to the longest oscillation length,

$$
l_{21} \equiv \frac{4 \pi E}{\delta m_{21}^{2}}=2.48 \times 10^{4} \mathrm{~km} \cdot\left(\frac{E}{\mathrm{GeV}}\right) \cdot\left(\frac{\delta m_{21}^{2}}{10^{-4} \mathrm{eV}^{2}}\right)^{-1} .
$$

The value of $\rho$ in eq.(24) is the average value of the crust and mantle, allowing that $L=10000 \mathrm{~km}$ can be realized without touching core of the Earth.

The graphs of $\tilde{J}$ as a function of energy, both perturbed and full calculation results, is also displayed in Fig. 3 and Fig. 6 .

We observe in the figures that our approximation works well, and as we stated both the T-violation effect and $\tilde{J}$ has resonant peak. The T-violation effect gets up over $10 \%$ for some case even $\omega$ is considerably small thanks to the resonant peak of $\tilde{J}$, leaving chance to search T-violation effect for such small- $\omega$ region.

Note that not only $\tilde{J}$ but also summation of three $\sin \left(\delta \tilde{m}_{i j}^{2} L\right) /(2 E)$ 's must be large in order to obtain large T-violation effect. Thus the peak of $\mathrm{T}$ violation may shift a little from $E_{\text {peak }}$.

\subsection{The limits of the T-violation search in terms of $\sin ^{2} 2 \omega$ and $\delta m_{21}^{2}$}

We consider here limits of parameter region we can cover in search of $\mathrm{T}$ violation. We will give limits in terms of $\delta m_{21}^{2}$ and $s_{2 \omega}$ so that we can refer to the diagram of the solar neutrino drawn in $\sin ^{2} 2 \theta-\delta m^{2}$ plane.

We first pay attention to the behavior of $\tilde{J}$ near $E=E_{\text {peak }}$ referring eq.(14). The half-width $\Delta a$ of the resonance of $\sin \tilde{\omega}$ can be estimated from

$$
\Delta a \cdot c_{\phi}^{2}=\delta m_{21}^{2} s_{2 \omega}
$$

The half-width in terms of the neutrino energy, $\Delta E$, is thus given by

$$
\begin{aligned}
\Delta E & =\frac{\delta m_{21}^{2}}{2 \sqrt{2} G_{\mathrm{F}} n_{\mathrm{e}}} \frac{s_{2 \omega}}{c_{\phi}^{2}} \\
& =294 \mathrm{MeV} \times \frac{s_{2 \omega}}{c_{\phi}^{2}}\left(\frac{\delta m_{21}^{2}}{10^{-4} \mathrm{eV}^{2}}\right)\left(\frac{\rho}{4.46 \mathrm{~g} \mathrm{~cm}-3}\right)^{-1} .
\end{aligned}
$$

The resonance gets sharper and narrower as $\sin 2 \omega$ gets smaller. We can pick up the resonance peak if the width is large compared the energy resolution $\delta E$. The condition $\Delta E \geq \delta E$ leads to

$$
\delta m_{21}^{2} s_{2 \omega} \geq 10^{-4} \mathrm{eV}^{2} \cdot c_{\phi}^{2}\left(\frac{\rho}{4.46 \mathrm{~g} \mathrm{~cm}^{-3}}\right)\left(\frac{\delta E}{294 \mathrm{MeV}}\right) .
$$



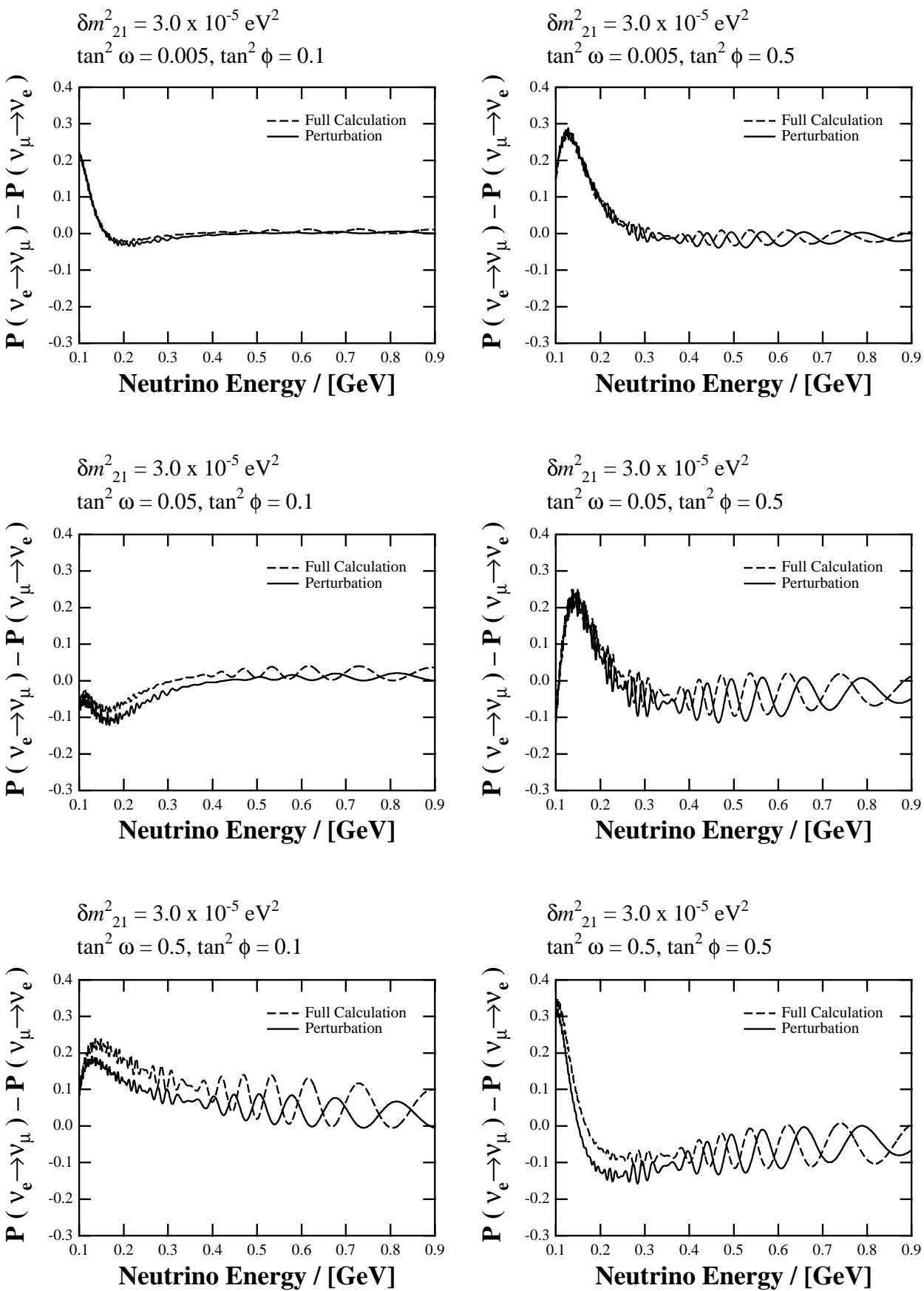

Figure 1: The approximated oscillation probabilities (solid lines) compared with unapproximated ones (dashed lines). The parameters not indicated in the figures are taken same as eqs.(24). Some parameter sets shown here are not favored by the solar neutrino observations, but we have presented them together to show the tendency of the graphs. 

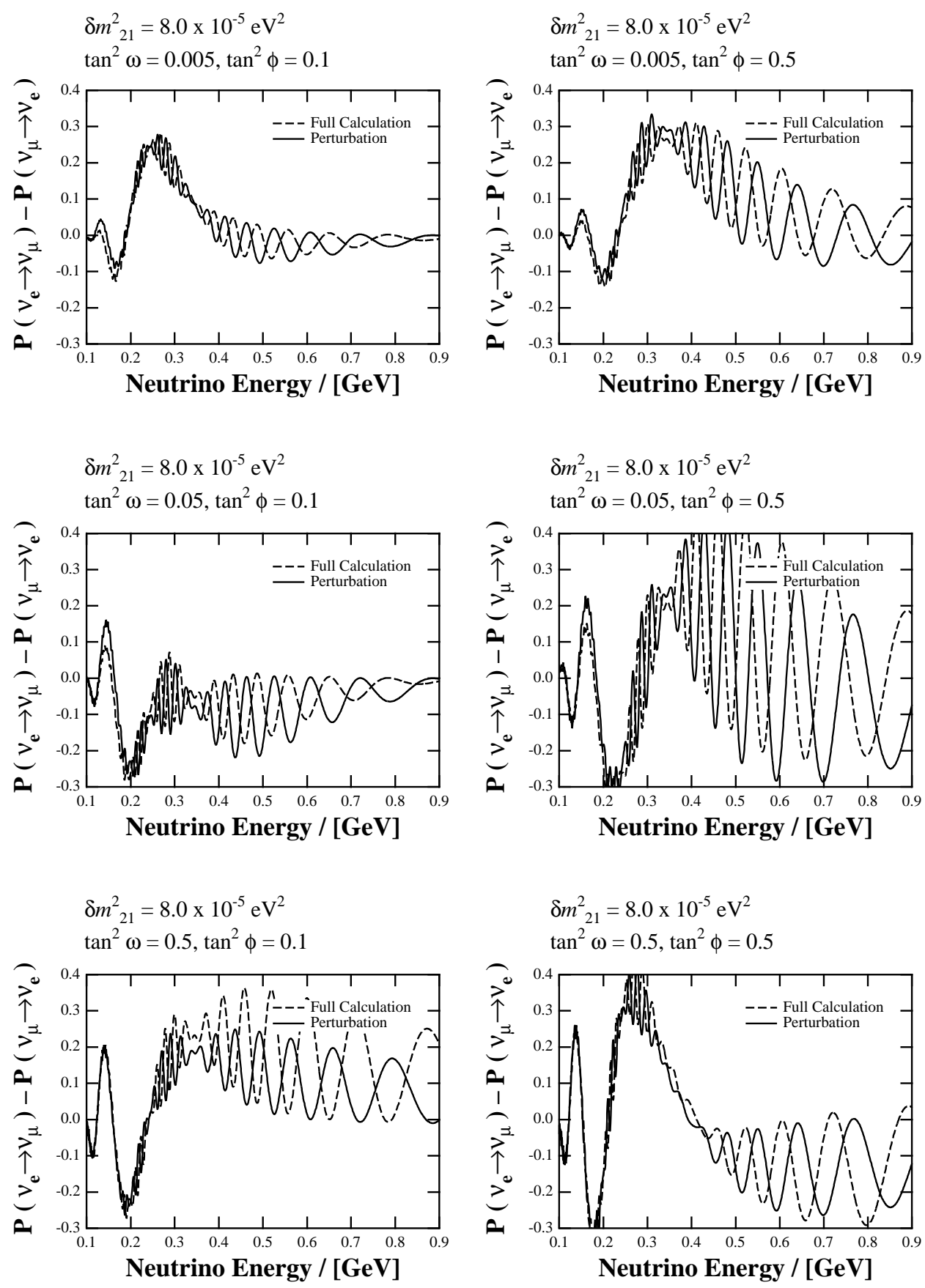

Figure 2: Same as Fig.2, but $\delta m_{21}^{2}=8 \times 10^{-5} \mathrm{eV}^{2}$. 

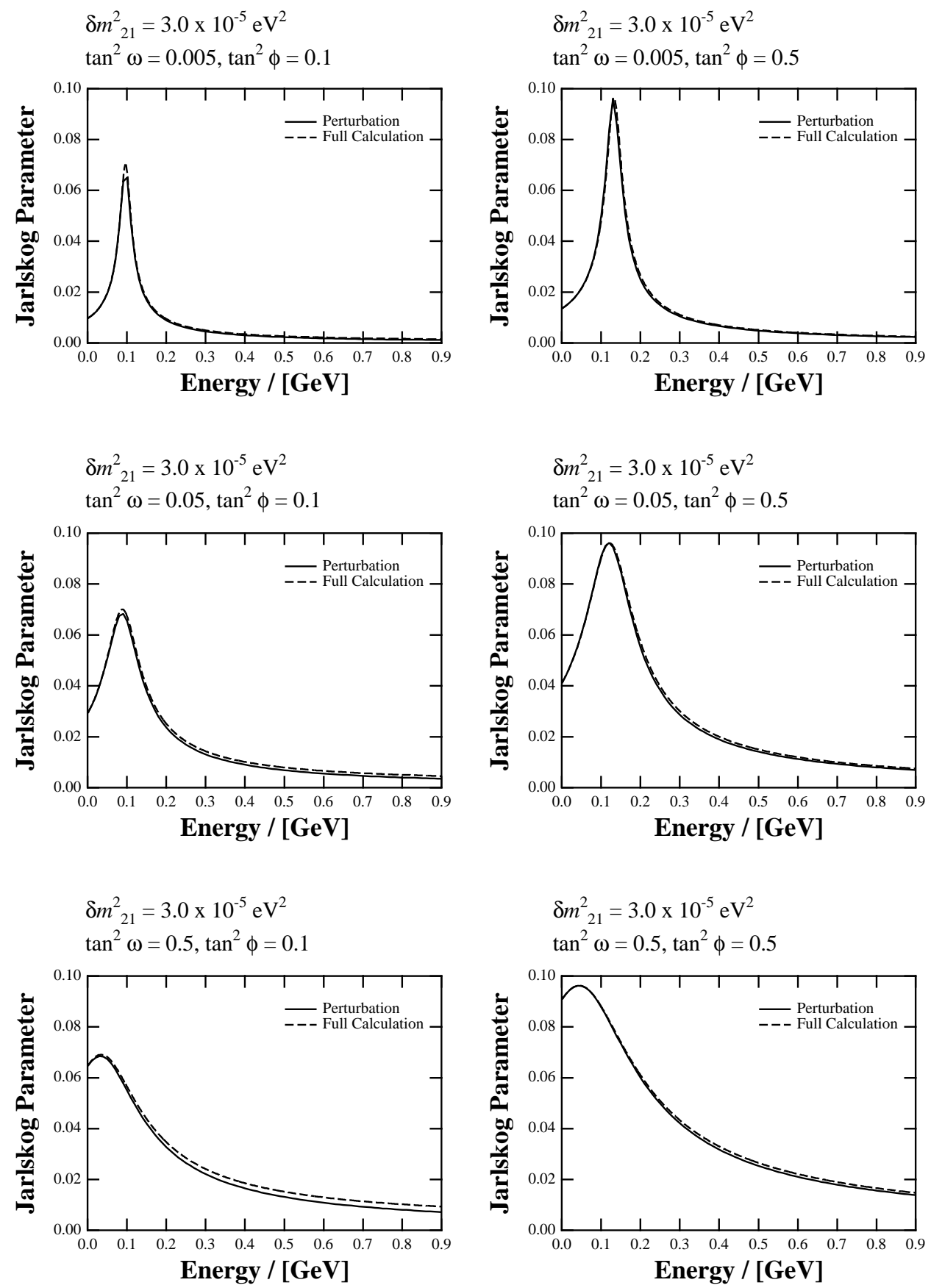

Figure 3: Effective Jarlskog parameter in the matter as a function of neutrino energy. Parameters are taken same as in Fig.1. 

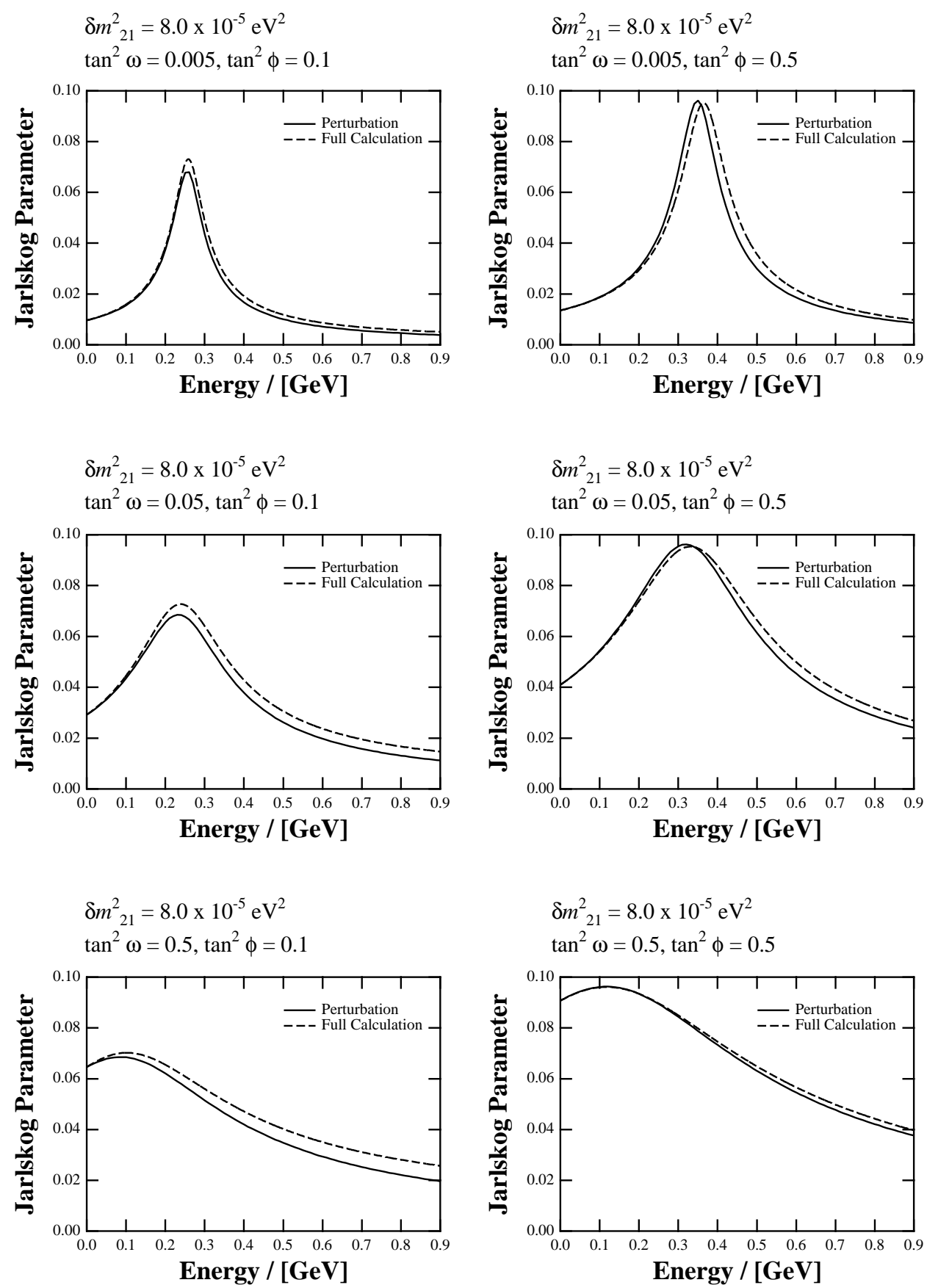

Figure 4: Same as Fig. [3, but parameters are same as in Fig.1. 
As the width gets smaller than the resolution the observed $T$ violation effect will be averaged over outside the peak, so we can make less use of the resonance. Thus the smaller energy resolution enables to reach smaller $\omega$ region in search of T-violation effect.

There is another limit in searching small $\omega$ region. We need

$$
\frac{\delta \tilde{m}_{21}^{2} L}{2 E} \gtrsim 1 \text { at } E=E_{\text {peak }}
$$

so that we make use of the resonance peak. Equation (29) leads to

$$
\frac{2 \pi L}{l_{21}} s_{2 \omega} \gtrsim 1
$$

or, with eqs. (15), (16) and (25),

$$
\delta m_{21}^{2} s_{2 \omega} \gtrsim 0.39 \times 10^{-4} \mathrm{eV}^{2} \cdot\left(\frac{E}{\mathrm{GeV}}\right)\left(\frac{L}{10000 \mathrm{~km}}\right)^{-1} .
$$

Almost all part of large MSW mixing angle solution can be thus covered with this result. Note that one can reach still smaller value by taking the tail of the peak at $E=E_{\text {peak }}$. For instance, the limit in case one observe T-violation effect at $E=E_{\text {peak }} \pm \Delta E$ is

$$
\delta m_{21}^{2} s_{2 \omega} \gtrsim 0.28 \times 10^{-4} \mathrm{eV}^{2} \cdot\left(\frac{E}{\mathrm{GeV}}\right)\left(\frac{L}{10000 \mathrm{~km}}\right)^{-1} .
$$

In Fig.5 and Fig.6 we show how the oscillation length $\tilde{l}_{21}$ changes as a function of neutrino energy calculated from the perturbation result.

\section{Summary and discussion}

We have considered the possibility of searching $T$ violation by the very long baseline neutrino oscillation experiments. The matter on the baseline effectivly changes only the mixing angle of the first-second generation. The effective Jarlskog parameter has an resonant peak at $\sim O(100 \mathrm{MeV})$ and also $\mathrm{T}$ violation effect grows by resonance. Making use of the resonance, the wider region in $\delta m_{21}^{2}-s_{2 \omega}^{2}$ plane can be covered in the search of $\mathrm{T}$ violation. The limits of search is given by the energy resolution and baseline length. Almost all part of the large MSW mixing angle solution can be searched with $L \sim 10000 \mathrm{~km}$ and $E \sim O(100 \mathrm{MeV})$.

\section{References}

[1] For a review, M. Fukugita and T. Yanagida, in Physics and Astrophysics of Neutrinos, edited by M. Fukugita and A. Suzuki (Springer-Verlag, Tokyo, 1994).

[2] GALlEX Collaboration, W. Hampel et al., Phys. Lett. B 447, 127 (1999) . 

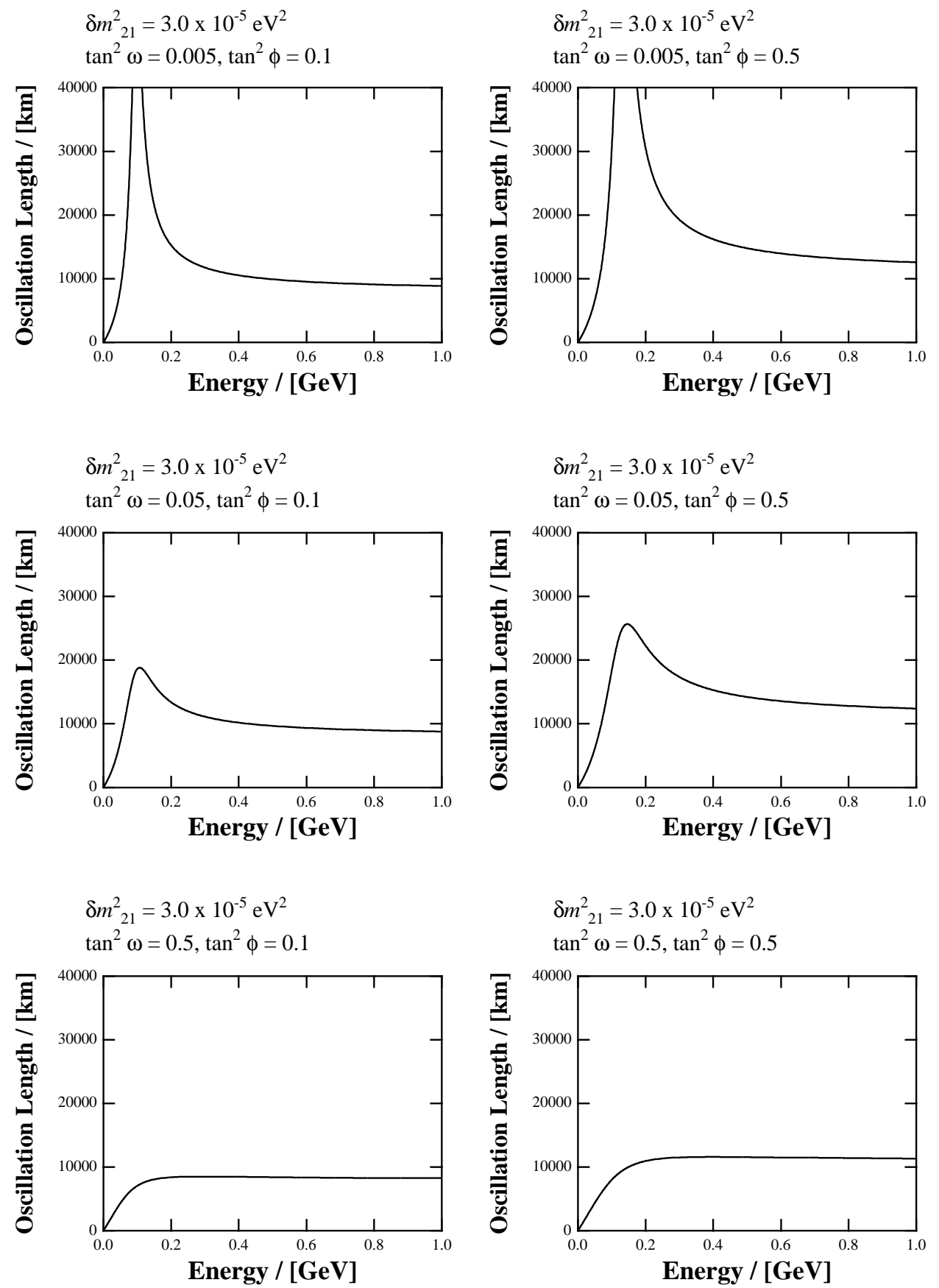

Figure 5: The longest oscillation length $\tilde{l}_{21}$ in the matter as a function of neutrino energy. The graph is calculated from the perturbated results. Parameters are taken same as in Fig. 

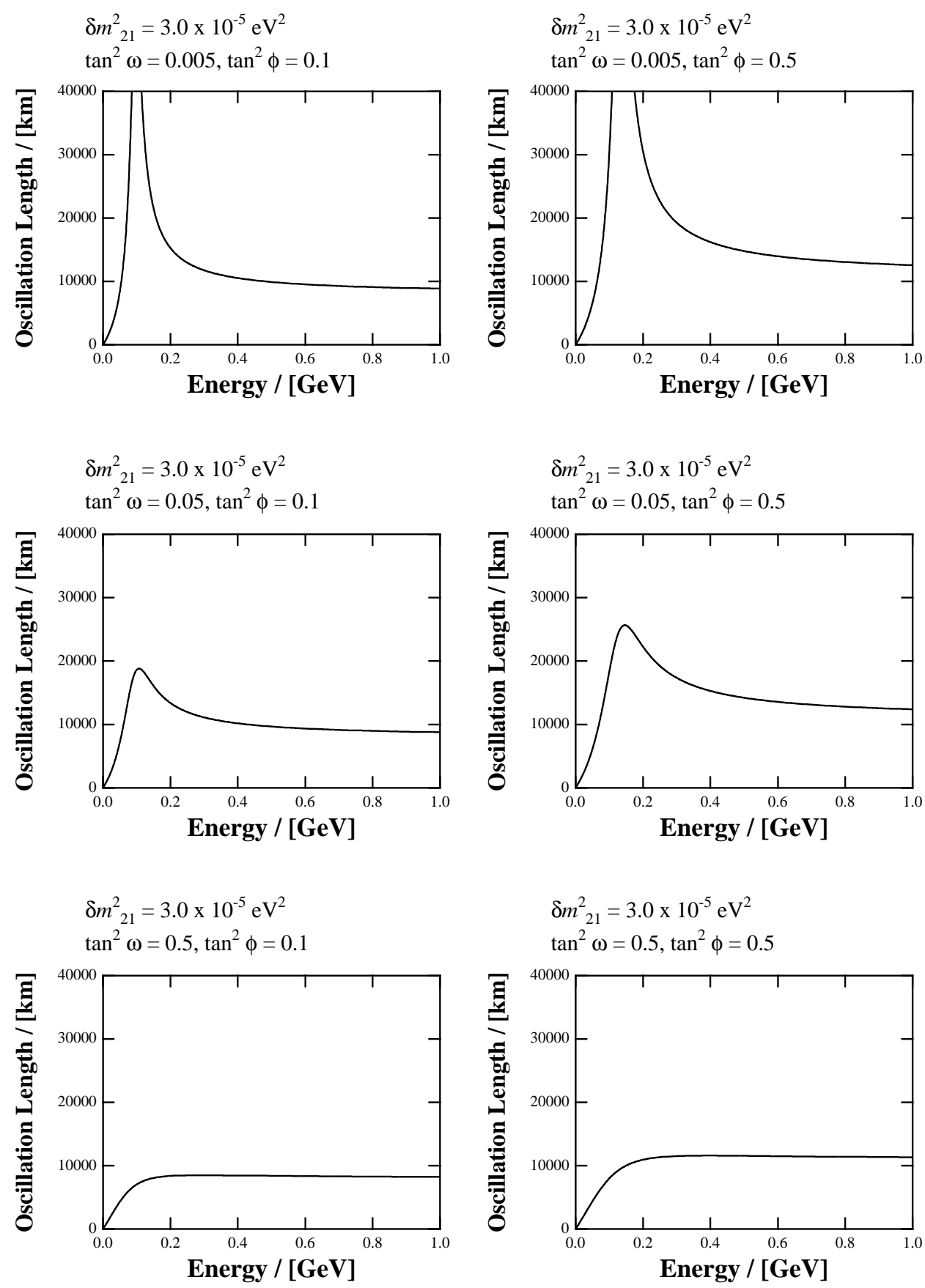

Figure 6: Same as Fig.6, but parameters are same as in Fig.1. 
[3] SAGE Collaboration, J. N. Abdurashitov et al., astro-ph/9907113.

[4] Kamiokande Collaboration, Y. Suzuki, Nucl. Phys. B (Proc. Suppl.) 38, 54 (1995).

[5] Homestake Collaboration, B. T. Cleveland et al., Astrophys. J. 496, 505 (1998)

[6] Super-Kamiokande Collaboration, Y. Fukuda et al., Phys. Rev. Lett. 82,1810 (1999), ibid. 82, 2430 (1999).

[7] Kamiokande Collaboration, K. S. Hirata et al., Phys. Lett. B205,416 (1988); ibid. B280,146 (1992); Y. Fukuda et al., Phys. Lett. B335, 237 (1994).

[8] IMB Collaboration, D. Casper et al., Phys. Rev. Lett. 66, 2561 (1991); R. Becker-Szendy et al., Phys. Rev. D46, 3720 (1992).

[9] SOUDAN2 Collaboration, T. Kafka, Nucl. Phys. B (Proc. Suppl.) 35, 427 (1994); M. C. Goodman, ibid. 38, 337 (1995); W. W. M. Allison et al., Phys. Lett. B391, 491 (1997).

[10] MACRO Collaboration, M. Ambrosio et al., Phys. Lett. B434, 451 (1998).

[11] Super-Kamiokande Collaboration, Y. Fukuda et al., Phys. Rev. Lett. 81, 1562 (1998), Phys. Lett. B433, 9 (1998), Phys. Lett. B436, 33 (1998), Phys. Rev. Lett. 82,2644 (1999).

[12] LSND Collaboration, C.Athanassopoulos et al., Phys. Rev. Lett 77, 3082 (1996); ibid 81, 1774 (1998).

[13] M. Tanimoto, Phys. Rev. D 55, 322 (1997); Prog. Theor. Phys.97, 901 (1997).

[14] J. Arafune and J. Sato, Phys. Rev. D55, 1653 (1997).

[15] J. Arafune, M. Koike and J. Sato, Phys. Rev. D56, 3093 (1997).

[16] H. Minakata and H. Nunokawa, Phys. Rev. D57 4403 (1998); Phys. Lett. B413, 369 (1997).

[17] M. Bilenky, C. Giunti, W. Grimus, Phys. Rev. D58, 033001 (1998).

[18] A.M. Gago, V. Pleitez and R. Zukanovich Funchal, hep-ph/9810505 .

[19] R. Barbieri, P. Creminelli and A. Romanino, hep-ph/9903460.

[20] O. Yasuda, hep-ph/9910428.

[21] V. Berger, S.Geer and K. Whisnant, to appear in Phys. Rev. D (hep$\mathrm{ph} / 9906478)$.

[22] M. Tanimoto, hep-ph/9906516; A. Kalliomki, J. Maalampi, M. Tanimoto, hepph/9909301.

[23] A. Romanino, hep-ph/9909425.

[24] A. De Rujula, M. B. Gavela and P. Hemandez, Nucl. Phys. B547, 21 (1999); A. Donini, M. B. Gavela, P. Hemandez and S. Rigolin, hep-ph/9909254.

[25] Y. Kuno and Y. Mori, Talk at the ICFA/ECFA Workshop "Neutrino Factories based on Muon Storage Ring", July 1999; Y. Kuno, in Proceedings of the Workshop on High Intensity Secondary Beam with Phase Rotation. 
[26] M. Koike and J.Sato, hep-ph/9909469 to appear in Phys. Rev. D.

[27] N. Cabibbo, Phys. Lett. B72, 333 (1978).

[28] V. Barger, K. Whisnant and R. J. N. Phillips, Phys. Rev. Lett. 45, 2084 (1980).

[29] L. Wolfenstein, Phys. Rev. D17, 2369 (1978).

[30] S. P. Mikheev and A. Yu. Smirnov, Sov. J. Nucl. Phys. 42, 913 (1985).

[31] G.L. Fogli, E. Lisi, and D. Montanino, Astropart. Phys. 9, 119 (1998).

[32] T. K. Kuo and J. Pantaleone, Phys. Lett. B 198, 406 (1987).

[33] P. I. Krastev and S. T. Petcov, Phys. Lett.B205, 84 (1988).

[34] S. Toshev, Phys. Lett. B 226, 335 (1989).

[35] S. M. Bilenky and S. T. Petcov, Rev. Mod. Phys. 59, 671 (1987).

[36] S. Pakvasa, in High Energy Physics - 1980, Proceedings of the 20th International Conference on High Energy Physics, Madison, Wisconsin, edited by L. Durand and L. Pondrom, AIP Conf. Proc. No. 68 (AIP, New York, 1981), Vol. 2, pp. 1164 .

[37] Z. Maki, M. Nakagawa and S. Sakata, Prog. Theor. Phys. 28, 870 (1962).

[38] L. -L. Chau and W. -Y. Keung, Phys. Rev. Lett. 59, 671 (1987). 
$\tan ^{2} \omega=0.005, \tan ^{2} \phi=0.1$
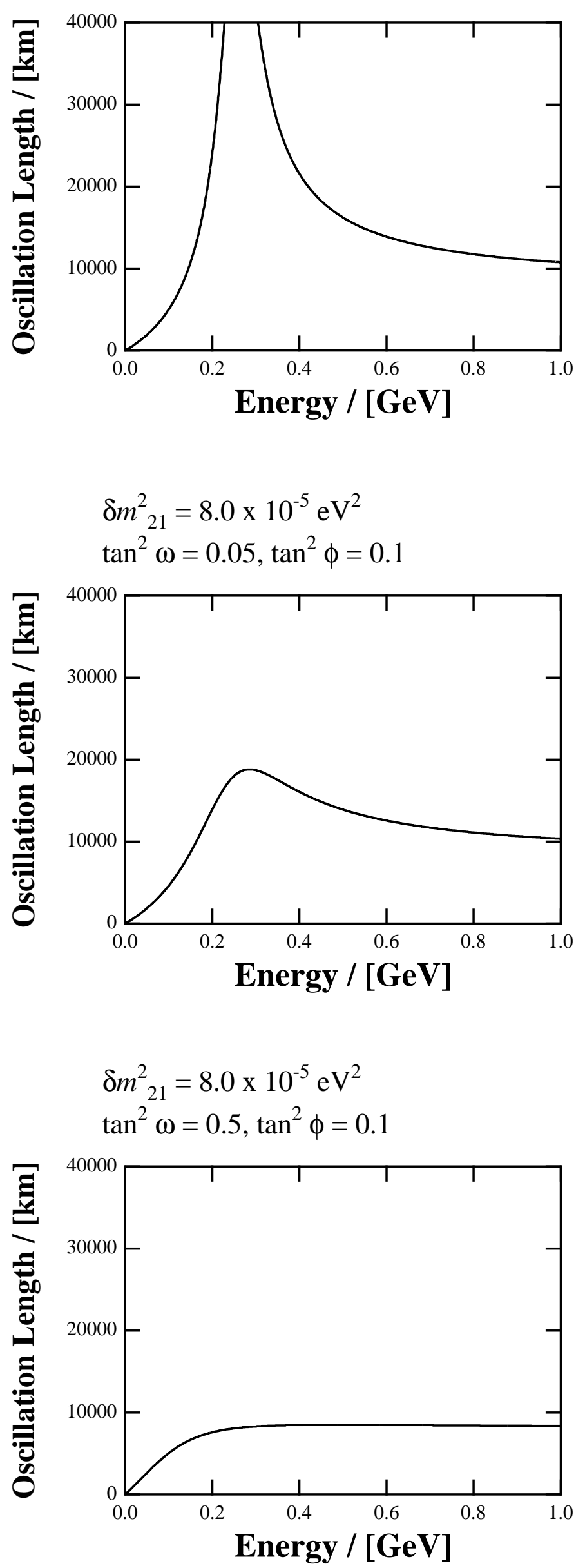
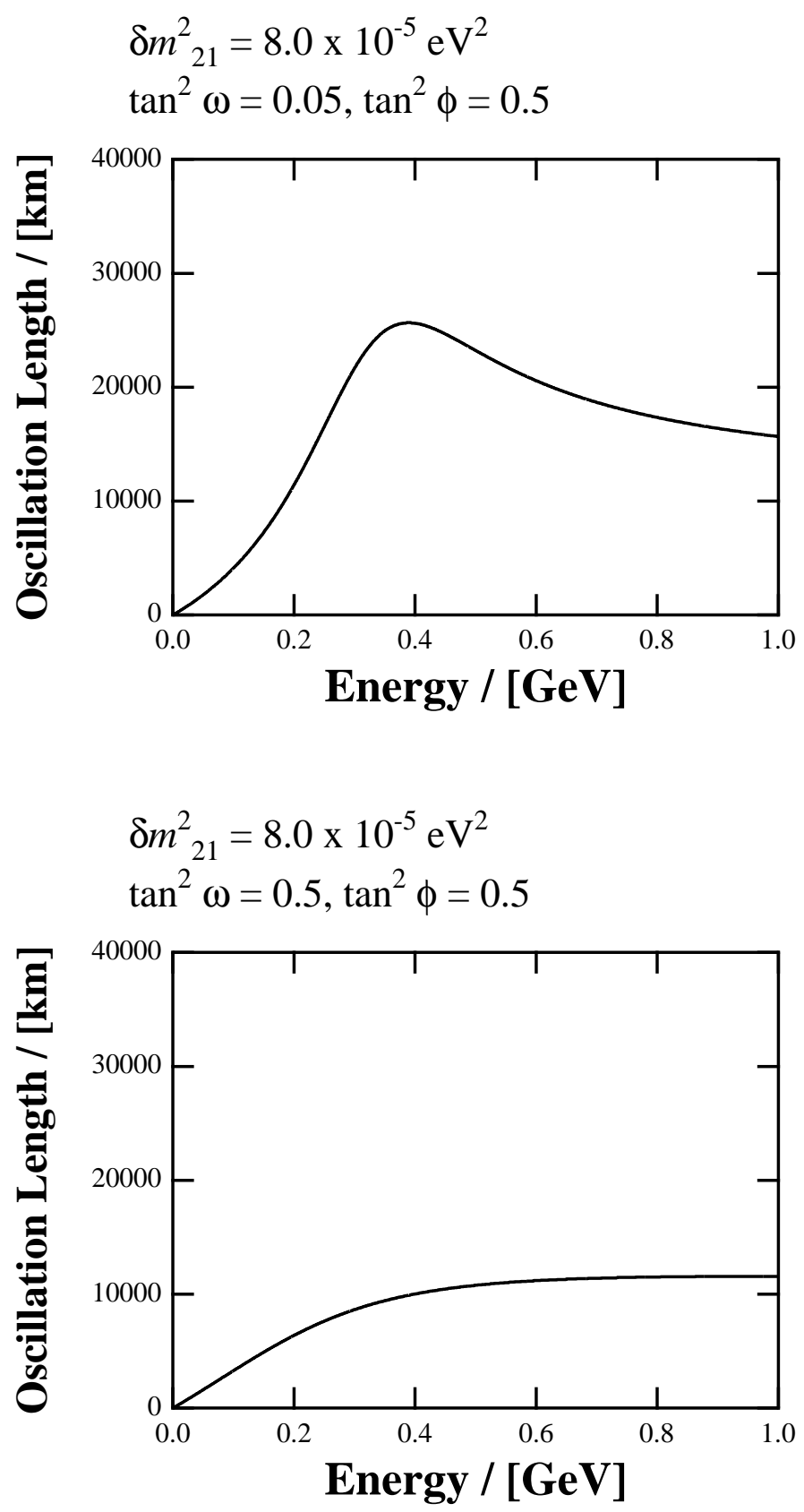